Accepted on 27 Nov 2014, for publication in Vacuum, Elsevier

\title{
Chromium Coated Silicon Nitride Electron Beam Exit Window
}

\section{N. Manivannan (corresponding author)}

Electronic and Computer Engineering, Brunel University, Uxbridge, UB8 3PH, UK

Email: Nadarajah.Manivannan@ @runel.ac.uk Tel: +44 (0) 1895265950

W. Balachandran

Electronic and Computer Engineering, Brunel University, Uxbridge, UB8 3PH, UK

Email: wamadeva.balachandran@brunel.ac.uk

C. Ribton

TWI Ltd, Granta Park, Gt Abington, Cambridge CB21 6AL, UK

Email: colin.ribton@twi.co.uk

\section{R. Beleca}

Electronic and Computer Engineering, Brunel University, Uxbridge, UB8 3PH, UK

Email: radu. beleca@brunel.ac.uk

\section{Abbod}

Electronic and Computer Engineering, Brunel University, Uxbridge, UB8 3PH, UK

Email: maysam. abbod @brunel.ac.uk

M. Cox

Silson Ltd, J B J Business Park, Northampton Rd, Northampton NN7 3DW, UK

Email: malcolm.cox@silson.com

P. Anastasia

Silson Ltd, J B J Business Park, Northampton Rd, Northampton NN7 3DW, UK

Email: peter.anastasi@silson.com

Abstract: $\mathrm{A} \mathrm{Si}_{3} \mathrm{~N}_{4}$ membrane with a thin $\mathrm{Cr}$ coating is proposed and demonstrated as an electron beam exit window. On average, $85 \%$ electron power transmission efficiency was achieved with a $1 \mu \mathrm{m}$ thick 
$\mathrm{Si}_{3} \mathrm{~N}_{4}$ membrane coated with $1 \mu \mathrm{m}$ thick $\mathrm{Cr}$ and the membrane sustained a beam current of up to $3 \mathrm{~mA}$ at $60 \mathrm{keV}$ electron energy for the continuous operation of 3 minutes. However, for an uncoated membrane of same thickness, the average electron power transmission efficiency was $71 \%$ and the maximum beam current sustained was $800 \mu \mathrm{A}$. It was also shown that a one micron thick $\mathrm{Si}_{3} \mathrm{~N}_{4}$ square membrane window of $10 \mathrm{~mm} \times 10 \mathrm{~mm}$ could withstand a differential pressure of 1.3 bars.

Key words: Electron Beam Windows; Electron Beam Processing; Thin Membrane; Metal Coating

Electron gun accelerators are used to produce electron beams for numerous applications [1]; to produce plasma, to weld metals, to melt materials, to treat gas [2-4], to treat bio waste [5], to treat waste water [6], to modify surfaces [7] and to make micro/nano patterns on materials [8]. Many of these applications require the electron beam to be used in atmospheric pressure whereas the electron beam is generated in vacuum for any reasons such as efficient accelerating, better beam shaping, avoid plasma formation, and to protect thermionic emitters from chemical reactions. In such cases, an Electron Exit Window (EEW) is used to transfer electron beam from vacuum into atmosphere as shown in Fig.1. A good EEW should be transparent to electrons and a strong barrier between atmospheric pressure and vacuum.

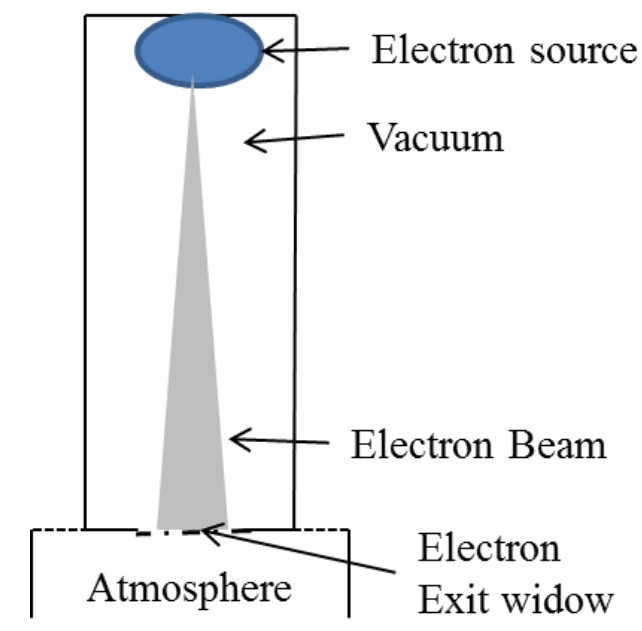

Fig. 1. Electron Beam Generation

Thin film membranes (e.g.: Silicon, Silicon Nitride and Titanium) are proposed in literature and used in industry in low power applications such as TEM and SEM [9-10]. Relatively thick (up to $100 \mu \mathrm{m}$ ) Aluminium foil has been widely used in industry for high electron energy (up to $6 \mathrm{MeV}$ ) applications [11-12]. Reported power transmission efficiencies of the EEW are between $13 \%$ and $95 \%$, this being mainly dependent upon the material, its thickness, and the electron energy. In the case of [11], a large Al foil window was used and the electron beam was scanned across the area and operated in 
pulsed mode operation $(0.375 \mu$ s pulses and duty cycle is less than $1 \%)$ to avoid the thermal loading and consequence failure of the $\mathrm{Al}$ foil. Furthermore, a complex cooling technique was required to take the heat away from the window membrane. It was shown in [12] that Al coated polyester film improved the power transmission efficiency by a factor of two compared to just $\mathrm{Al}$ alone; however the duration for which the foil was exposed to the electron beam was very low $(20 \mu \mathrm{s})$ and duty cycle was much less than 1\%. Electron gun based plasma generation for flue gas treatment (e.g.: deNOx and deSOx) $[2,4]$ requires a high beam current with low electron energy in order to maintain a high dosage (e.g.: 9kGy) in the plasma. In such cases, the power efficiency of the system was very low $(<50 \%)$ mainly due to inefficient electron transmission of power through EEW. This means electron guns need to operate at more than $2 \times$ more power than actually required to compensate the power loss at EEW. This makes the plasma treatment of flue very expensive both (capital and operational) and environmentally harmful. This study is aimed at low electron energy $(\sim 60 \mathrm{keV})$ beams to generate non-thermal plasma as very low mean electron energy $(1-5 e \mathrm{e})$ is only required to treat exhaust gas [13] and use of high (> $1 \mathrm{MeV})$ or medium $(0.5$ to $1 \mathrm{MeV})$ electron energy is an overkill. Low electron energy would also give many other benefits, such as reduced cost in terms of capital cost (voltage supply, electron beam column and associated accessories) and running cost (lower electrical energy, longer life of electron source and less cooling), smaller foot print and eco-friendly. Furthermore, the absolute power loss at EEW will be much less for $60 \mathrm{keV}$ than high/medium energy electron beam and therefore the required EEW cooling is much less.

The two main underlying criteria of an EEW are electron power transmission efficiency (EPTE) and the ability to withstand the differential pressure. These two criteria have opposing requirement on the thickness of a membrane; i.e.: the thinner the EEW the higher EPTE, however, lower the ability to withstand differential pressure and vice versa. The major challenge in using a thin film window is the heat generated due to electron collision with the window material. The thinner the window the better the electron transmission and hence less heat dissipation and vice versa. However, a thinner window means its thermal capacity is less and therefore the risk of damaging the material is very high.

In this paper, a preliminary study of the chromium coated $\mathrm{Si}_{3} \mathrm{~N}_{4} \mathrm{EEW}$ is reported. The major reasons for the choice of $\mathrm{Si}_{3} \mathrm{~N}_{4} \mathrm{EEW}$ were manufacturability to a fraction of a micron thickness and its superior mechanical properties. $\mathrm{Si}_{3} \mathrm{~N}_{4}$ has high yield strength $(14 \mathrm{Gpa})$, superior thermal shock resistance $(>600 \sigma \mathrm{K})$, good oxidation resistance, low thermal expansion $\left(3.3 \times 10^{-6} / \mathrm{K}\right)$ and high temperature range (melting point: $2660 \mathrm{~K}-2770 \mathrm{~K}$ ). The limiting properties of $\mathrm{Si}_{3} \mathrm{~N}_{4}$ were low thermal conductivity (5-30 
$\mathrm{W} / \mathrm{m} \mathrm{K})$ and low electrical conductivity $\left(10^{-13}-10^{-8} \mathrm{~S} / \mathrm{m}\right)$. High electrical conductance is needed in order to remove any accumulated charge in the membrane to ground. To overcome these challenges, $\mathrm{Cr}$ coating was applied to the $\mathrm{Si}_{3} \mathrm{~N}_{4} \mathrm{EEW}$. Cr has relatively good thermal conductance $(94 \mathrm{~W} / \mathrm{m} \mathrm{K})$ and good electrical conductance $\left(7.9 \times 10^{6} \mathrm{~S} / \mathrm{m}\right)$.

In this preliminary study, six $\mathrm{Si}_{3} \mathrm{~N}_{4}$ membranes (LPCVD deposited silicon rich nitride) were manufactured; half of them were coated with $\mathrm{Cr}$ of $\mu \mathrm{m}$ thickness. A simple heat sink was also designed and adopted as a means of heat transfer from the EEW to the atmosphere. The schematic and photograph of the $\mathrm{Si}_{3} \mathrm{~N}_{4}$ EEW with $\mathrm{Cr}$ coating and associated heat sink is shown in Fig. 2.
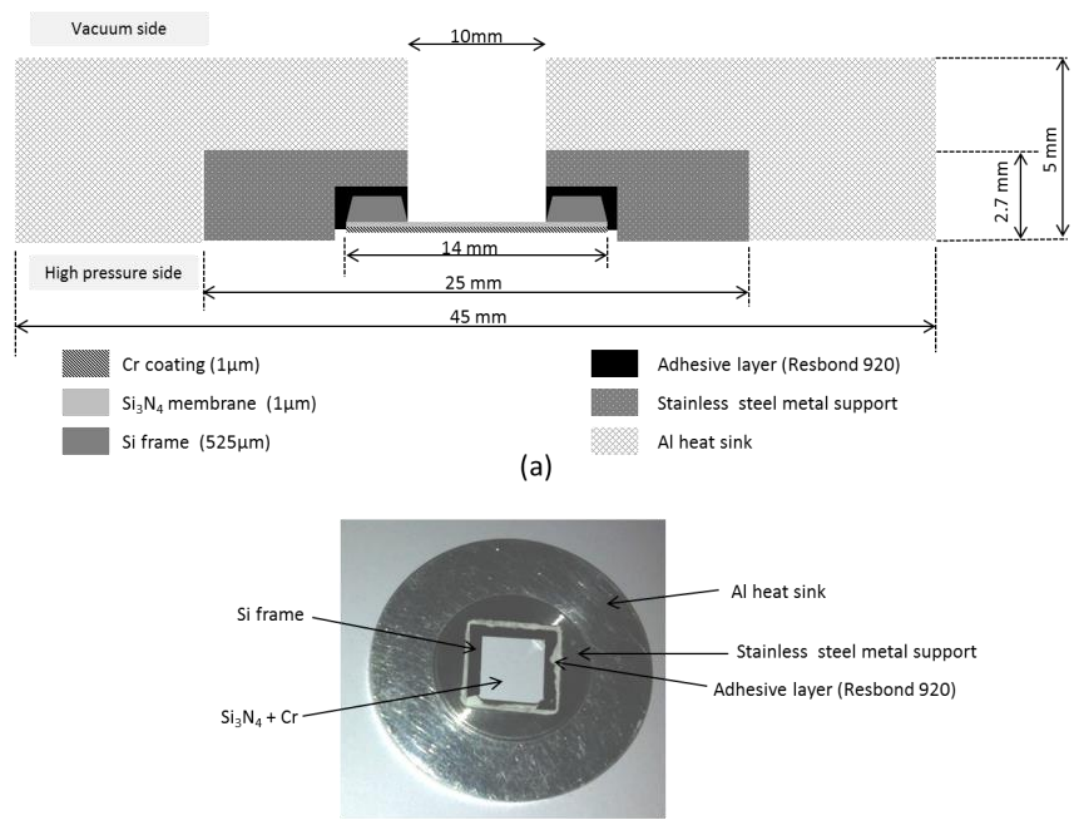

(b)

Fig. 2. $\mathrm{Si}_{3} \mathrm{~N}_{4} \mathrm{EEW}$ with Cr coating (a) $2 \mathrm{D}$ Schematic (b) Photograph

FEM models of the $\mathrm{Si}_{3} \mathrm{~N}_{4}$ EEW (with and without $\mathrm{Cr}$ ) coating were developed and solved in COMSOL multi-physics platform. This model was used to simulate heat transfer by conduction and radiation. The following equation of heat transfer by conduction was used to solve for temperature, $T[\mathrm{~K}]$, in the solids.

$$
\rho C_{p} \frac{\partial T}{\partial t}=\nabla \cdot(k \nabla T)+Q
$$


Where $\rho$ density of the material $\left[\mathrm{kg} / \mathrm{m}^{3}\right], C_{p}$ is the specific heat capacity $[\mathrm{J} /(\mathrm{kg} \cdot \mathrm{K})], k$ is thermal conductivity of the material $[\mathrm{W} /(\mathrm{kg} . \mathrm{K})]$, and $Q$ is heat source other than viscous heating[W/m $\left.{ }^{3}\right]$. In our case $\mathrm{Q}$ is the energy lost by the electron beam.

The equation governing the heat radiation (derived from Stefan-Bolzman law) to solve for temperature is;

$$
-n .(-k \nabla T)=\varepsilon \rho\left(T_{a}^{4}-T^{4}\right)
$$

where $n$ is normal vector to the plane of radiating surface, $\varepsilon$ is the emissivity of material and $\mathrm{T}_{\mathrm{a}}$ is ambient temperature.

A set of typical results obtained from the computer simulation \{for the schematic shown in Fig. 2(a) \} are shown in the Fig. 3 for the temperature of $\mathrm{Si}_{3} \mathrm{~N}_{4}$ when the power deposited on the membrane was $25 \mathrm{~W}$. As shown in Fig. 3(a), the membrane reached a steady-state temperature for the cases of uncoated and coated with $\mathrm{Cr}$. However, $\mathrm{Cr}$ coated showed superior performance over uncoated; the final steady-state temperature was $15 \%$ lower and the temperature gradient was also much lower, i.e. it took $50 \mathrm{~ms}$ to reach the steady-state temperature, while it was $20 \mathrm{~ms}$ for the uncoated membrane. A lower temperature gradient can allow the material to heat up slowly and hence reduced the risk of heat shock. Fig. 3(b) shows the temperature profile of the integrated EEW structure (Fig. 1). As shown, the temperature of the EEW was much higher than everything else as it is bound to be. The temperature started to drop at the edge of the membrane and the rate of decrease is much higher in uncoated than $\mathrm{Cr}$ coated. This is another benefit of the Cr coating as it reduces the likelihood of the fracture at the edges. Finally Fig. 3(c) shows the 3D profile of the temperature of the Cr coated window system. This figure also clearly shows a ring at the edge of the membrane, which indicates the high temperature drop at the edge of the EEW.

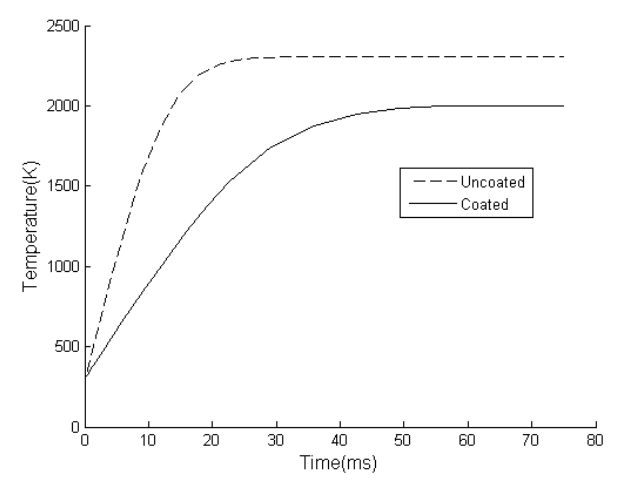




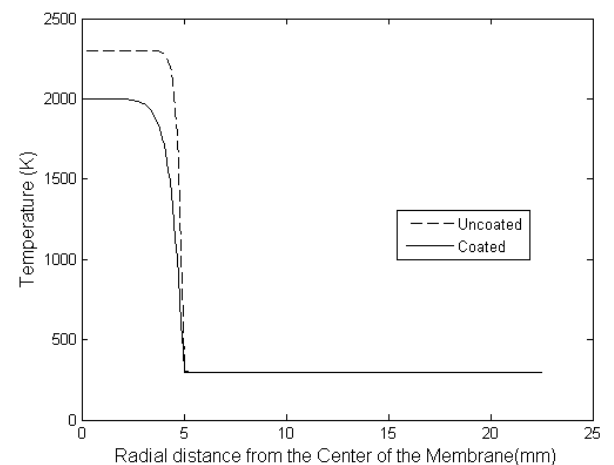

(b)

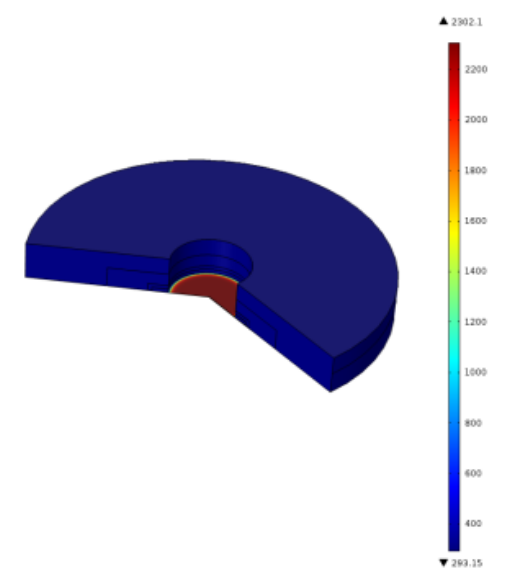

FIG.3. Temperature profile of $\mathrm{Si}_{3} \mathrm{~N}_{4}$ membrane from FEM simulation for power deposition of $25 \mathrm{~W}$

(a) Temperature vs time

(b) Temperature vs radial distance from the centre of membrane and

(c) 3D temperature profile with Cr coating. 
Two sets of electron power transmission experiments were performed for $\mathrm{Si}_{3} \mathrm{~N}_{4} \mathrm{EEW}$, with and without $\mathrm{Cr}$ coating using an electron beam gun. The electron gun facilities available at TWI Ltd were used for these experiments and the experiments were performed in vacuum. The beam current after passing through the EEW was measured using a Faraday cup, which was connected to an ammeter. An electron beam of $60 \mathrm{keV}$ was used in both experiments while beam current was adjusted from a low value (10uA) to higher values (a few mA) until the membrane failed.

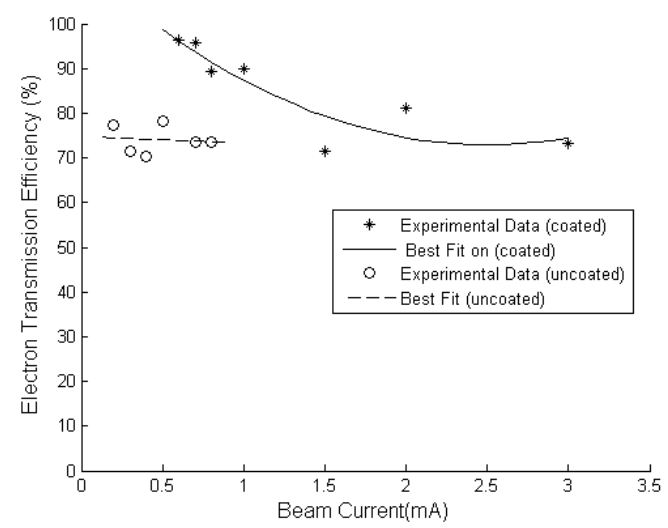

(a)

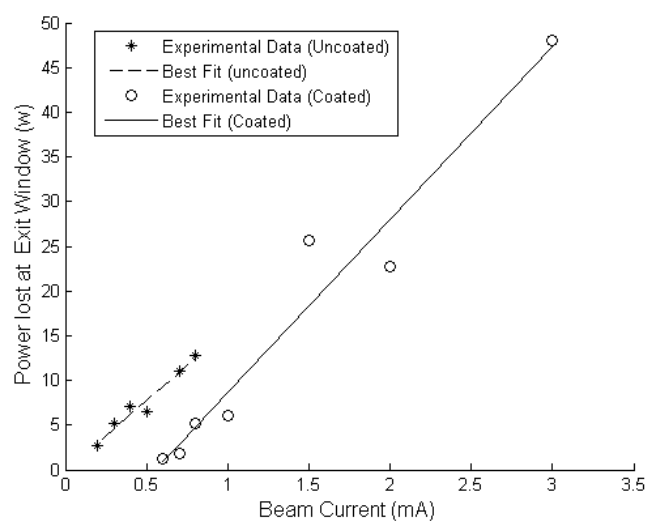

(b)

FIG.4: Experimental results (a) electron transmission efficiency (b) power lost at the membrane

Fig. 4 shows the results obtained from the experiments. Fig. 4(a) shows the electron power transmission efficiency of uncoated and coated $\mathrm{Si}_{3} \mathrm{~N}_{4}$ and Fig. 4(b) shows the power lost in the $\mathrm{Si}_{3} \mathrm{~N}_{4}$ EEW. The electron power transmission efficiency was calculated as the percentage ratio between the 
detected current with and without the $\mathrm{Si}_{3} \mathrm{~N}_{4} \mathrm{EEW}$. It was assumed that electron energy loss while transmitting through the EEW was very minimal due to the small thickness and ignored in these calculations.

The uncoated $\mathrm{Si}_{3} \mathrm{~N}_{4}$ EEW allowed $75 \%$ of electron power to pass through and sustained a continuous beam current of up to $0.8 \mathrm{~mA}$. Beyond this current the membrane was damaged. $\mathrm{The} \mathrm{Cr}$ coated $\mathrm{Si}_{3} \mathrm{~N}_{4}$ EEW sustained beam currents of up to $3 \mathrm{~mA}$. The electron power transmission efficiency of the coated membrane was very high ( $>90 \%$ ) at low beam current and dropped significantly for higher beam current. As can be seen in Fig. 4(b), more power is lost at high beam current. This lost power was turned into heat in the membrane material and had increased the vibrations of the atoms in the membrane material. Therefore, it was very likely to have more collisions between the transmitted electrons and the atoms of the membrane; thus resulted in significant decrease in electron transmission through the EEW. However, a Cr coating has enabled an increase in the transmitted beam current by a factor of four because of improved heat conduction. Furthermore, it should be noted that transmitted electrons scattered at large angles when passing through the EEW may not have been collected by the Faraday cup and hence the presented results on transmission efficiency are underestimated.

A series of simulations was performed using a COMSOL FEM model to investigate the maximum power that could be deposited in the $\mathrm{Si}_{3} \mathrm{~N}_{4}$ membrane (coated and uncoated) by calculating the temperature distribution and considering the highest temperature sustainable before the membrane would become damaged. The first benefit of the coating is that the rate of increase of temperature is slow with respect to power loss with a coated membrane compared to that of uncoated. For example, the uncoated $\mathrm{Si}_{3} \mathrm{~N}_{4}$ membrane reached a steady-state temperature of $2000 \mathrm{~K}$ with just below $15 \mathrm{~W}$ of heat deposition whereas the $\mathrm{Cr}$ coated counterpart required $25 \mathrm{~W}$ to reach the same steady-state temperature. The second advantage of the coating is that it allowed the membrane to maintain higher heat power loss and sustained higher temperature before failure. The uncoated membrane was damaged when heat power loss is $\sim 15 \mathrm{~W}$, whereas a coated one withstood the heat power loss close up to $50 \mathrm{~W}$. These results are manifested in the calculation of the maximum sustainable beam current as shown in Fig. 4(a).

Another set of experiments were performed to investigate the ability of the $\mathrm{Si}_{3} \mathrm{~N}_{4}$ membrane to withstand differential pressure. Fig. 5(a) shows the experimental set-up used to carry out the tests. The chamber below the membrane was kept at one bar and the pressure on the other side of the membrane was increased in steps for one minute duration. The maximum differential pressure (burst pressure) recorded 
was the last pressure for which the membrane survived for a complete minute. Figure 5(b) shows the scatter diagram of burst pressure of $10 \times 10 \mathrm{~mm} \mathrm{Si}_{3} \mathrm{~N}_{4}$ membrane for the thicknesses up to $1 \mu \mathrm{m}$. These results show that $1 \mu \mathrm{m}$ thickness $\mathrm{Si}_{3} \mathrm{~N}_{4}$ membrane can withstand differential pressure up to 1.3 bars, which is more than the atmospheric pressure. The additional test carried out for the $\mathrm{Si}_{3} \mathrm{~N}_{4}$ with $\mathrm{Cr}$ coating showed the burst pressure of 2 bars, which is mainly due to the increased thickness of the coated window. Note all these pressures were $25 \%$ less than actual pressures measured in the experiments to allow an error margin in practical applications.

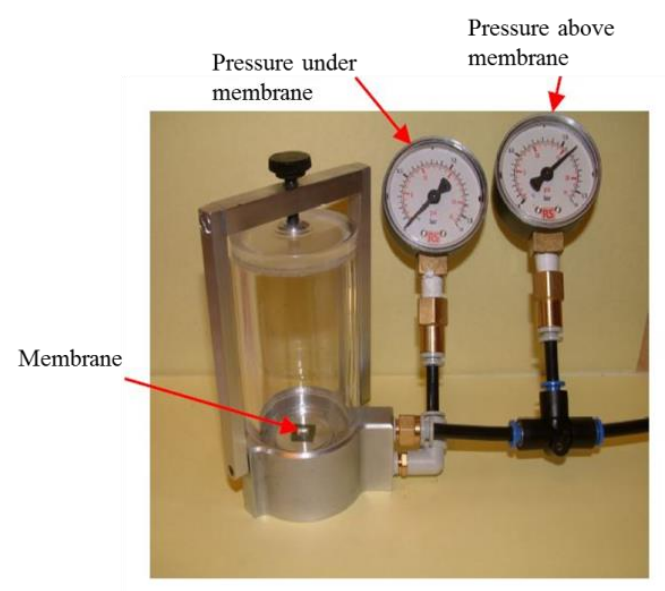

(a)

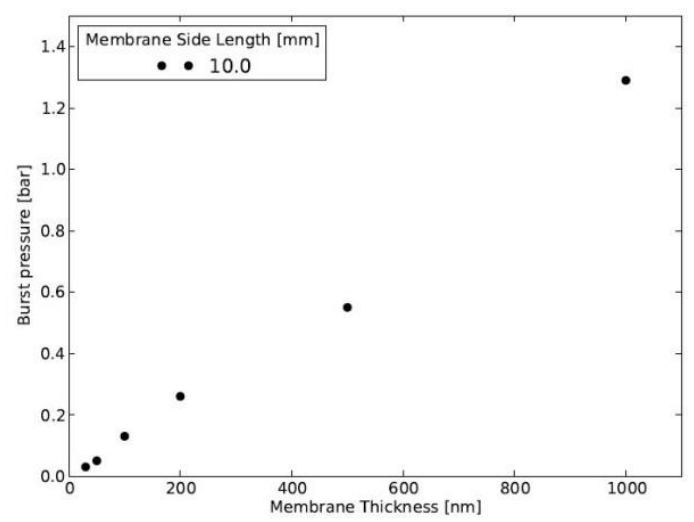

(b)

Fig. 5. Burst Pressure Test (a) Experimental setup (b) Results (Pressure Vs Size)

In summary, A FEM model simulating heat dissipation and electron transmission experimental results show that coating $\mathrm{Si}_{3} \mathrm{~N}_{4}$ membrane with a thermally conductive material $(\mathrm{Cr})$ has improved the electron transmission capability of an electron beam exit window. While $\mathrm{Si}_{3} \mathrm{~N}_{4}$ has the ability to 
withstand high differential pressures and has a high melting point, $\mathrm{Cr}$ coating acts as good thermal conducting medium good electrical conductor.

Further research need to be carried out to understand the suitability of $\mathrm{Si}_{3} \mathrm{~N}_{4}$ membrane based electron beam exit windows to successfully operate at high beam currents (10-100mA) and higher electron energies $(60-100 \mathrm{keV})$. This would require systematic investigation of various material coatings, both sides of coating and their thickness; noting the fact that metal coating on the vacuum side would assist the collided electrons to be conducted away and avoid charge accumalation. Max burst pressure measurements with coated $\mathrm{SI}_{3} \mathrm{~N}_{4}$ will also be carried out. It will also important to investigate the possibility of pulsed electron beam operations to keep the electron exit window for longer life (few good months). A theoretical model relating temperature of the membrane with electron transmission efficiency will also another important area of future research.

The work carried out at Brunel University was co-funded by the EC Seventh Framework Programme theme FP7-SST-2011-RTD-1 for the DEECON project (grant number 284745).

\section{References}

[1] Cleland MR. CERN Yellow Report 2006; 2006-012.

[2] Martin M, Margaritescu I, Cirstea E, Togoe I, Ighigeanu D. Vacuum 2005; 77; 501- 6.

[3] Chmielewski AG, Sun Y, Pawelec A, Licki J. Catalysis Today 2012; 191; 159- 64.

[4] Chmielewski AG. Radiation Physics and Chemistry 2007; 76 ; 1480- 84.

[5] Chmielewski A, Sun Y, Bułka S, Zimek Z. Radiation Physics and Chemistr 2007; 76; 1795- 801.

[6] Kim TH, Lee JK, Lee MJ. Radiation Physics and Chemistry 2007; 76;1037- 41.

[7] Zou J, Zhang K, Hao S, Dong C and Grosdidier T. Thin Solid Films 2010; 519( 4);1404- 15.

[8] Withers F, Bointon TH, Dubois M, Russo S and Craciun MF. Nano Letters 2011; 11( 9); 3912- 6.

[9] Nishiyama H, Suga M, Ogura T, Maruyama Y, Koizumi M. Journal of Structural Biology 2010; 169; 438- 49 .

[10] Dougal RA, Liu S. Journal of Vacuum Science and Technology B 2000;18; 2750- 6.

[11] Ighigeanu D, Martin D, Calinescu I, Manaila E, Craciun G. Proceeding of the seventh Nuclear and Particle Physics Conference (NUPPAC) 2009;42;447-59. 
[12] Chalise PR, Watanabe M, Okino A, Hotta E, KO KC. Digest of Technical Papers, PPC-2003, $14^{\text {th }}$ IEEE International $2003 ; 1 ; 275-8$.

13 McAdams R, J. Phys. D: Appl. Phys. 2001;34; 2810-2821. 